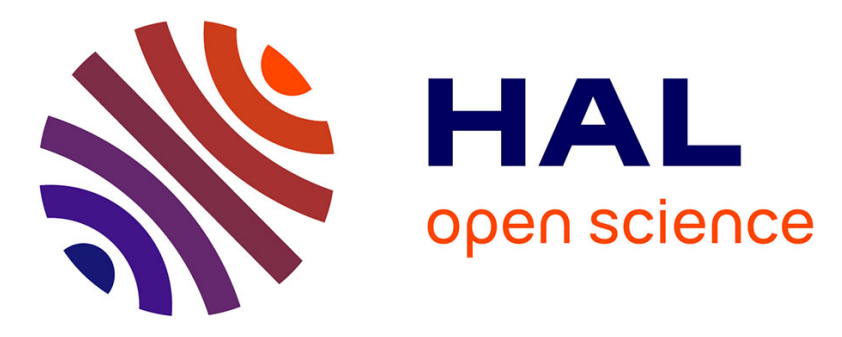

\title{
Gene expression profile predicts outcome after anthracycline-based adjuvant chemotherapy in early breast cancer
}

François Bertucci, Nathalie Borie, Henri Roche, Thomas Bachelot, Jean-Marc Doussal, Gaëtan Macgrogan, Stéphane Debono, Agnès Martinec, Isabelle Treilleux, Pascal Finetti, et al.

\section{To cite this version:}

François Bertucci, Nathalie Borie, Henri Roche, Thomas Bachelot, Jean-Marc Doussal, et al.. Gene expression profile predicts outcome after anthracycline-based adjuvant chemotherapy in early breast cancer. Breast Cancer Research and Treatment, 2010, 127 (2), pp.363-373. 10.1007/s10549-010-1003z . hal-00594464

\section{HAL Id: hal-00594464 https://hal.science/hal-00594464}

Submitted on 20 May 2011

HAL is a multi-disciplinary open access archive for the deposit and dissemination of scientific research documents, whether they are published or not. The documents may come from teaching and research institutions in France or abroad, or from public or private research centers.
L'archive ouverte pluridisciplinaire HAL, est destinée au dépôt et à la diffusion de documents scientifiques de niveau recherche, publiés ou non, émanant des établissements d'enseignement et de recherche français ou étrangers, des laboratoires publics ou privés. 


\title{
Gene expression profile predicts outcome
}

after anthracycline-based adjuvant chemotherapy in early breast cancer

\author{
François BERTUCCI ${ }^{1,2,3}$, Nathalie BORIE ${ }^{4}$, Henri ROCHE ${ }^{5}$, \\ Thomas BACHELOT ${ }^{6}$, Jean-Marc LE DOUSSAL ${ }^{4}$, Gaëtan MACGROGAN ${ }^{7}$, Stéphane \\ DEBONO ${ }^{4}$, Agnès MARTINEC $^{4}$, Isabelle TREILLEUX ${ }^{8}$, \\ Pascal FINETTI ${ }^{1}$, Benjamin ESTERNI ${ }^{9}$, Jean-Marc EXTRA ${ }^{2}$, \\ Jean GENEVE ${ }^{10}$, Fabienne HERMITTE ${ }^{4}$, Christian CHABANNON ${ }^{11}$, \\ Jocelyne JACQUEMIER ${ }^{1,12}$, Anne-Laure MARTIN ${ }^{10}$, Michel LONGY ${ }^{13}$, \\ Dominique MARANINCHI ${ }^{14}$, Vincent FERT ${ }^{4}$, \\ Daniel BIRNBAUM ${ }^{1}$, Patrice VIENS ${ }^{2,3}$
}

Short title : Genomics of breast cancer and survival after adjuvant chemotherapy

\section{Addresses:}

1. Centre de Recherche en Cancérologie de Marseille, Département d'Oncologie Moléculaire, UMR891 Inserm; Institut Paoli-Calmettes (IPC) ; IFR137, Marseille, France,

2. Département d'Oncologie Médicale, IPC, Marseille, France,

3. Université de la Méditerranée, Marseille, France,

4. Ipsogen S.A., Marseille, France,

5. Département d’Oncologie Médicale, Institut Claudius Régaud, Toulouse, France,

6. Département d'Oncologie Médicale, Centre Léon Bérard, Lyon, France,

7. Département d'Anatomopathologie, Institut Bergonié, Bordeaux, France,

8. Département d’Anatomopathologie, Centre Léon Bérard, Lyon, France,

9. Bureau d'Etudes Cliniques et Thérapeutiques, IPC, Marseille, France, 
10. Bureau d'Etudes Cliniques et Thérapeutiques, Fédération Nationale des Centres de Lutte contre le Cancer, Paris, France,

11. Centre de Ressources Biologiques en Oncologie, IPC, Marseille, France,

12. Département de BioPathologie, IPC, Marseille, France,

13. Laboratoire de Génétique Moléculaire, Institut Bergonié, Bordeaux, France.

14. Institut National du Cancer, Paris, France.

Corresponding author: Pr François BERTUCCI: Département d'Oncologie Médicale,

UMR891 Inserm, Institut Paoli-Calmettes, 232, bd Sainte-Marguerite, 13273 Marseille, France.

Phone: 334912235 37; Fax : 334912236 70;

E-mail: bertuccif@marseille.fnclcc.fr 


\section{ABSTRACT}

Purpose. Prognosis of early beast cancer is heterogeneous. Today, no histo-clinical or biological factor predictive for clinical outcome after adjuvant anthracycline-based chemotherapy (CT) has been validated and introduced in routine use. Using DNA microarrays, we searched for a gene expression signature associated with metastatic relapse after adjuvant anthracycline-based CT without taxane.

Methods. We profiled a multicentric series of 595 breast cancers including 498 treated with such adjuvant $\mathrm{CT}$. The identification of the prognostic signature was done using a metagene-based supervised approach in a learning set of 323 patients. The signature was then tested on an independent validation set comprised of 175 similarly treated patients, 128 of them from the PACS01 prospective clinical trial.

Results. We identified a 3-metagene predictor of metastatic relapse in the learning set, and confirmed its independent prognostic impact in the validation set. In multivariate analysis, the predictor outperformed the individual current prognostic factors, as well as the Nottingham Prognostic Index-based classifier, both in the learning and the validation sets, and added independent prognostic information. Among the patients treated with adjuvant anthracycline-based CT, with a median follow-up of 68 months, the 5-year metastasis-free survival was $82 \%$ in the "good-prognosis" group and $56 \%$ in the "poorprognosis" group.

Conclusion. Our predictor refines the prediction of metastasis-free survival after adjuvant anthracycline-based CT and might help tailoring adjuvant CT regimens. 


\section{KEY WORDS}

Adjuvant chemotherapy, breast cancer, DNA microarray, genomics, prognosis.

\section{LIST OF ABBREVIATIONS}

CT: chemotherapy; IPC: Institut Paoli-Calmettes; CLB: Centre Léon Bérard; IB: Institut Bergonié; SBR: Scarff-Bloom-Richardson; IHC: immunohistochemistry; ER: estrogen receptor; PR: progesterone receptor; FEC: 5-fluoro-uracile + epirubicine + cyclophosphamide; PCR: polymerase chain reaction; MFS: metastasis-free survival; HR: hazard ratio. 


\section{INTRODUCTION}

Survival of early breast cancer patients has increased in relation with screening programs and adjuvant hormone therapy (HT), chemotherapy (CT) [1], and trastuzumab monoclonal antibody [2-4]. Benefits of CT were first demonstrated in the 1970s with the CMF (cyclophosphamide, methotrexate, fluorouracil) regimen, then with the addition of anthracycline in the 1980s [5]. During the last decade, 4-6 cycles of anthracycline plus cyclophosphamide +/- fluorouracil have been used as "standard" adjuvant regimens for axillary lymph node-negative $(\mathrm{N}-)$ and positive $(\mathrm{N}+)$ patients [6]. Taxanes were introduced in the anthracycline-based regimens for $\mathrm{N}+$ patients in the late 2000s [7].

The factors used in routine to predict the metastatic risk and the probability of therapeutic response are insufficient to face the heterogeneous clinical behavior of breast cancer. In consequence, all $\mathrm{N}+$ patients empirically receive regimens based on anthracyclines and taxanes without knowing if the tumor will be sensitive to this drug combination [8]. Taxanes are expensive, toxic, and the associated benefit is small ( 3 to $7 \%)$ in term of 5-year survival [9-13], suggesting that a majority of patients do not benefit from the addition of taxane to anthracycline-based regimens. Thus, taxanes are not universally accepted as standard treatment [7], and an anthracycline-based regimen without taxane remains a valid option. Today, the choice of adjuvant cytotoxic regimen is based more upon the risks of relapse and toxicity, patient's comorbidities, and physician's experience, than upon the probability of efficiency. Given the increasing availability of new drugs, orienting patients towards the most likely beneficial regimen is even more crucial. This challenge supposes to better assess the metastatic risk after a 
given CT regimen. Today, no biological factor predictive for efficiency of anthracyclinebased adjuvant CT has been validated and introduced in routine use $[14,15]$.

Several retrospective studies have shown the prognostic potential of gene expression profiling in breast cancer [16]. Relevant subtypes of disease have been identified $[17,18]$. Multigene signatures associated with survival have been reported in different settings: without any adjuvant systemic therapy [19-28], and after adjuvant HT [29-33]. Two of them are being tested in prospective randomized genomics-based trials launched in N- patients [34]: "Microarray in Node-Negative Disease May Avoid Chemotherapy Trial" (MINDACT), and "Trial Assigning Individualized Options for Treatment (Rx)" (TAlLORx), the aim of which is to decrease the unnecessary indications of adjuvant CT. Some of these prognostic tests are commercialized and proposed to help the management of $\mathrm{N}$ - patients [35]. By contrast, few data are available on the signatures specifically associated with clinical outcome after CT. Some studies analyzed the response to primary CT [36-44]. One reported a signature specific to the delivered regimens [40]. In the setting of adjuvant CT, the published series are relatively small [45-48] or heterogeneous regarding CT regimens $[49,50]$.

In this study, we aimed at identifying a signature predictive of metastatic relapse after adjuvant anthracycline-based CT without taxane. Using DNA microarrays, we profiled a multicentric series of 595 breast carcinomas, including 498 from patients treated with such adjuvant regimen. To avoid overfitting often pointed out in microarray studies, the predictor was first identified in a learning set of 323 patients, then validated in an independent set of 175 patients treated for most of them in a multicentric prospective clinical trial [13]. 


\section{MATERIALS AND METHODS}

\section{Breast tumor samples}

Tumor samples were obtained after initial surgery from 595 early breast cancer patients treated in several French institutions: Institut Paoli-Calmettes (IPC, n=243), Centre Léon Bérard (CLB, $n=110$ ), Institut Bergonié (IB, $n=114$ ), and PACS01 clinical trial ( $A$ arm, $n=128$ ). Immediately after surgery, samples had been dissected by pathologists and snap-frozen in liquid nitrogen for storing.

Of these 595 tumors, 498 came from patients who had received adjuvant postoperative CT and were used to identify and validate the expression signature associated with metastatic relapse (Table 1). All had received 5 to 6 cycles of $2^{\text {nd }}$ generation adjuvant $\mathrm{CT}$, including an anthracycline (doxorubicine or epirubicine) combined with cyclophosphamide and fluorouracil. None of them had received taxane or trastuzumab in adjuvant setting. Patients with hormone receptor-positive tumor received adjuvant HT according to standard guidelines of the moment. They included all "IPC" and "CLB patients". The "IB samples", initially profiled for another study, included 17 cases treated with CT. Poor prognosis criteria that determined delivery of CT included $\mathrm{N}_{+}$ status or age under 40 years, pathological tumor size greater than $20 \mathrm{~mm}$, ScarffBloom-Richardson (SBR) grade equal to 3, or negative estrogen receptor status (ER) as evaluated by immunohistochemistry $(\mathbb{H C})$. The validation series also included 128 samples form $\mathrm{N}_{+}$patients treated in the PACS01 trial, a multicentric prospective randomized trial promoted by a network of national cancer centres (FNCLCC), which 
compared 6 FEC100 cycles (A arm) vs 3 FEC100 cycles followed by 3 docetaxel cycles ( $\mathrm{B}$ arm) for the adjuvant treatment [13]. In the A arm, 128 frozen tumor samples coming from 17 French hospitals were available for profiling. Histoclinical characteristics and outcome of these patients did not differ from the entire trial cohort. Follow-up data were obtained for all patients at regular intervals. The median follow-up of the 498-patient series was 68 months. During this period, 129 patients experienced metastatic relapse and the 5-year metastasis-free survival (MFS) was $76 \%$. The main characteristics of this series are listed in Table $2\left(1^{\text {st }}\right.$ and $2^{\text {nd }}$ columns). Before sending frozen samples to lpsogen, all tumor sections were reviewed by pathologists in each centre. All contained a percentage of cancer cells superior to $60 \%$. This study was approved by our local ethics committees, and all patients gave informed consent.

\section{Gene expression profiling and data analysis}

RNA extraction and gene expression profiling were done at lpsogen (Marseille, France). Profiling was done with two different platforms: platform 1 used lpsogen DiscoveryChip cDNA microarrays (lpsogen, Marseille, France; http://www.ipsogen.com/) for 565 samples, and platform 2 used Affymetrix U133 Plus 2.0 oligonucleotide chips for 30 samples from IPC. Platform 1 cDNA microarrays contained PCR products from a total of 9.040 Image and control clones. These microarrays were used as previously described [51-53]. After normalization, analysis was based on biologically and/or clinically relevant metagenes defined from supervised and unsupervised analyses. To prevent overfitting, the metagene-based predictor was identified in a learning set of 323 tumors out of the 498 samples treated with adjuvant 
$\mathrm{CT}$, and validated in the 175 remaining independent tumors. Experimental procedures and metagene-based data analysis are detailed in Supplementary Text.

\section{Statistical analyses}

Distributions of histoclinical factors were compared using the Chi-2 test. The primary end-point was the MFS, calculated from the date of diagnosis until the date of first distant metastasis. Data concerning patients without metastatic relapse at last follow-up were censored. Follow-up was calculated from the date of diagnosis to the time of last follow-up for censored patients. Survival curves were derived from KaplanMeier estimates [54] and compared by log-rank test. The prognostic impact of the genomic predictor was assessed in multivariate analysis by the Cox proportional hazard models, which included all features with a $15 \%$ level of significance in univariate analysis [55]. Cox models were built using a variable stepwise selection procedure. We also compared the prognostic value of our genomic classifier with that of the clinical Nottingham Prognostic Index (NPI) classifier [56]. Classical NPI cut-offs were used: score inferior or equal to 3.4 for low-risk, score between 3.5 and 5.4 for intermediaterisk, and score superior to 5.4 for high-risk. Survival rates and hazards ratios (HR) were calculated with their $95 \%$ confidence intervals $(95 \% \mathrm{Cl})$. Statistical tests were two-sided at the $5 \%$ level of significance.

\section{RESULTS}

\section{Identification and selection of metagenes}


To avoid complex steps of normalization between expression data generated from two different DNA microarray sources, this first part of the analysis was done from the data generated on platform 1 (565 samples).

\section{Identification of phenotypic signatures}

Supervised MaxT method identified five gene expression signatures associated with ER, PR, ERBB2, EGFR and Ki67 immunohistochemical (IHC) status of tumors, respectively. Results are summarized in Supplementary Table 1 (see Supplementary Table 2 for the complete gene lists). As expected, several genes previously associated with ER status were retrieved in the ER signature [45,57-59]: ESR1, GATA3, SCUBE2, TFF1, TFF3, and XBP1, overexpressed in the ER-positive samples, and STK6/AURKA, ENO1 and ITGB3, underexpressed. The ERBB2 signature contained genes located in the 17q amplicon (ERBB2, GRB7, ITGB3, PPARPB, PECAM1 and STAT3) [51,58].

The robustness of each signature was verified in the remaining tumors (Supplementary Table 1), with accuracy of prediction ranging from $78 \%$ to $96 \%$. From these five signatures, 10 gene subsets were defined (under- and over-ER, under- and over-PR, under- and over-ERBB2, under- and over-EGFR, and under- and over-Ki67), respectively composed by the genes positively and the genes negatively correlated with the phenotype. For example, the ER signature contained two subsets, "over-ER" and "under-ER", which included genes respectively overexpressed and underexpressed in ER-positive tumors. Then, for each subset, the expression levels of the component genes were averaged, defining a total of 10 metagenes.

\section{Hierarchical clustering}

Global clustering of the 468 tumors treated with adjuvant CT (Figure 1A) sorted 
two major classes of samples strongly correlated with the ER IHC status (Figure 1B): $85 \%$ of samples were ER-positive in the left class vs $32 \%$ in the right class $(p<0.0001$, Chi-2 test). Most tumors were grade III in the right class and grade I in the left class $(p<0.0001$, Chi-2 test). More tumors were ERBB2-positive in the right class than in the left class $(p<0.0001$, Chi-2 test). No correlation existed between clustering and metastatic relapse.

Clustering revealed clusters of co-expressed genes, some of which (Figure 1C) corresponding to defined biological processes or cell types $[37,58,60]$ : the "luminal", the "ERBB2", and the "basal" clusters, and other clusters ("proliferation", "stromal", "immune" and "early response"). We selected 17 gene clusters that satisfied to the chosen selection criteria (node correlation $\geq 0.5$ and minimum of 5 genes: see colored bars to the right of Figure 1A). Most of them were included in or were close to relevant above-cited gene clusters. For each subset, the corresponding metagene was computed as defined above.

\section{Selection of metagenes}

Twenty-seven gene subsets were thus defined: 10 from supervised analyses, and 17 from clustering. To avoid redundancy of variables (metagenes) entered in the Cox model, a two-step selection was applied. In a first step, 5 of the 17 subsets defined by clustering, which corresponded to phenotypic signatures, were excluded (Figure 1A). In a second step, colinearity analysis applied to subsets defined from supervised analyses excluded over-ER, over-PR and under-ERBB2, which were highly correlated with underEGFR and together. Eventually, 19 metagenes were retained for building the predictor.

\section{Identification of a genomic predictor associated with metastatic relapse}


Cox proportional hazards analysis was applied to these 19 metagenes entered as continuous variables (Supplementary Table 3) and to the 323 tumors of the learning set treated with adjuvant CT. Within this series, 98 patients developed metastatic relapse with a median time to relapse of 36 months, and 225 patients remained relapse-free with a median follow-up of 73 months. By univariate analysis, none of the metagenes was associated with MFS. By multivariate analysis, three metagenes (under-EGFR, under-PR and under-ER representing 189 genes/ESTs) were retained, suggesting a synergy between them. Genes/ESTs included in these three metagenes are listed in Supplementary Table 2. A "Risk Score" (RS) was computed as a linear combination of the three metagenes and calculated for each tumor as follows:

RS = (-2.90 x "under-ER") - (1.47 x “under-PR") - (4.17 x “under-EGFR")

The $\mathrm{RS}$ ranged from -1.33 to 1.37 , with a high score associated with poor outcome. The optimal cut-off was the $3^{\text {rd }}$ quartile (RS value=0.09). As expected, the predictor performed well in term of prognosis: the "good-prognosis group" (RS $<0.09$, 242 patients, 60 metastatic relapses) had a 5 -year MFS of $79 \%$, while the "poorprognosis group" (RS $>0.09,81$ patients, 38 metastatic relapses) had a 5 -year MFS of $52 \%(p<0.0001$, log-rank test) (Figure 2A). The hazard ratio $(\mathrm{HR})$ for metastatic relapse was $2.35(95 \% \mathrm{Cl}[1.6-3.5])$. The performances of this genomic predictor were compared with those of traditional individual prognostic features. As shown in Table 3, the genomic predictor was the most significant feature and remained significant in multivariate analysis, after adjustment for other features. 
We next tested our genomic predictor in the validation set of 175 independent samples (145 profiled using platform 1 and 30 using Affymetrix). None of these samples had been used to define the predictor. Of this series, 31 patients displayed a metastatic relapse with a median time of 30 months, and 144 women remained without metastatic relapse after a median follow-up of 61 months. The same "Risk Score", calculated for each patient, with the same threshold defined two groups in the same proportions as in the learning set $(75 \%$ and $25 \%$ respectively). These groups strongly correlated with outcome, suggesting the robustness of the predictor: 14 metastatic relapses in the 43 patients of the "poor-prognosis group" and only 17 in the 132 of the "good-prognosis group" ( $p=0.003$, Chi-2 test; Figure 2B). The accuracy of prediction of relapse was 74\%. The respective 5-year MFS were $87 \%$ and $65 \%$ ( $p<0.001$, log-rank test) with a HR of $3.18(95 \% \mathrm{Cl}[1.6-6.5])$. Respective MFS were $87 \%$ and $61 \%(p<0.001$, log-rank test) with a HR of $3.87(95 \% \mathrm{Cl}$ [1.6-9.0]) when applied only to the 128 patients of the prospective PACS01 trial.

Univariate and multivariate analyses were applied to this independent validation set (Table 3). Univariate analysis identified six factors as correlated with MFS $(p<0.05$, log-rank test): grade, number of positive axillary lymph nodes, pathological tumor size, ER and ERBB2 status, and the genomic predictor. In multivariate analysis, two factors remained significant, among which the predictor was the strongest one with an adjusted HR for metastasis of 4.5 for "poor-prognosis group" patients (95\%Cl [2.1-9.7]).

We compared the prognostic performances of the genomic predictor and the NPI classifier in the validation set. NPI score could be calculated for 166 out of 175 samples, with 7 cases defined as low-risk, 85 as intermediate-risk and 74 as high-risk. For reasons of size, we merged the low-risk and the intermediate-risk cases to create a "not 
high" risk group (92 cases) which was then compared to the high-risk group (74 cases). In univariate analysis, the NPI classifier was associated with MFS (Table 3). In multivariate analysis including the genomic and the NPI classifiers (Table 3) both remained significant with the genomic classifier showing the strongest HR (3.47 vs 2.44). Analysis of unadjusted and adjusted HR for MFS in high- versus low-risk patients as defined by the two classifiers confirmed these data. Adjustment of the genomic predictor HR for the NPI classifier increased from 3.2 to 3.5. By contrast, the HR of the NPI classifier decreased after adjustment for the genomic predictor from 2.8 to 2.5. The NPI low-risk patients ( $\mathrm{N}=92,90 \%$ 5-year MFS) were separated by the genomic predictor in "good-" and "poor-prognosis groups" with respective 5-year MFS of 93\% and $75 \%(p=0.02$, log-rank test; $\mathrm{HR}=4,95 \% \mathrm{Cl}[1.1-15.2])$. The same was true for the NPI high-risk patients ( $\mathrm{N}=74 ; 76 \%$ 5-year MFS) who were separated in "good-" and "poor-prognosis groups" with respective 5 -year MFS of $85 \%$ and $57 \%$ ( $p=0.009$, logrank test; $\mathrm{HR}=3.2,95 \% \mathrm{Cl}$ [1.3-8.2]). By contrast, the "good-prognosis group" defined by the genomic predictor ( $\mathrm{N}=123,90 \%$ 5-year MFS) was separated by the NPI classifier in "not high" and high risk groups with not significantly different 5-year MFS: 93\% and 85\% respectively ( $p=0.07$, log-rank test; $H R=2.7,95 \% \mathrm{Cl}[0.9-8.3])$. Similarly, he "poorprognosis" patients defined by the genomic predictor ( $N=43 ; 65 \% 5$-year MFS) were separated by the NPI classifier in two groups with not significantly different 5-year MFS $(p=0.15$, log-rank test; $\mathrm{HR}=2.3,95 \% \mathrm{Cl}[0.7-7.3])$.

\section{Genomic predictor-based classification and histoclinical correlations}

We then assessed the correlation of our predictor-based classification with histoclinical factors in the 498 pooled cases (Table 2). With a median follow-up of 68 
months after diagnosis, the 5-year MFS was 76\% (Supplementary Figure 1A). The factors associated with our classification ( $p<0.05$, Chi-2 test) were axillary lymph node status, SBR grade, ER, PR and Ki67 status. As expected, a strong correlation existed with clinical outcome: 52 of the 124 patients (42\%) assigned to the "poor-prognosis group" displayed metastatic relapse, vs only 77 of the $374(21 \%)$ assigned to the "goodprognosis group" ( $p<0.0001$, Chi-2 test). With similar follow-up, the respective 5 -year MFS were $56 \%$ and $82 \%(p<0.0001$, log-rank test; Supplementary Figure 1B). The HR for metastasis was $2.5(95 \% \mathrm{Cl}[1.8-3.6])$.

The genomic predictor kept its prognostic value in subgroups of patients. For $\mathrm{N}+$ patients (Supplementary Figure 1C), the 5-year MFS were $80 \%$ in the "goodprognosis group" and $54 \%$ in the "poor-prognosis group" ( $<<0.0001$, log-rank test). The same was true for $\mathrm{N}$ - patients, with respective 5 -year MFS of $95 \%$ vs $64 \%(p=0.005$, log-rank test; Supplementary Figure 1D). Interestingly, the 5-year MFS in the "goodprognosis" $\mathrm{N}+$ patients $(80 \%)$ was better than in the "poor-prognosis" $\mathrm{N}$ - patients $(64 \%$, $\mathrm{p}<0.05$; log-rank test). Similarly, in the ER-positive group, the 5-year MFS were $83 \%$ and $52 \%$ respectively $(p<0.0001, \log$-rank test). The same trend was observed for the ER-negative group with respective 5 -year MFS of $78 \%$ and $61 \%$ ( $p=0.12$, log-rank test). Unequal performance of a predictive multigene signature has been reported previously by others [33,61]. Histological grade is another important feature of breast cancer. Fiveyear MFS was $81 \%$ in the "good-prognosis group" and $49 \%$ in the "poor-prognosis group" within the grade II tumors $(p<0.001$, log-rank test). The same was true for the grade III tumors with respective 5 -year MFS of $77 \%$ and $57 \%$ ( $p=0.002$, log-rank test). These results showed that our predictor adds prognostic information beyond that provided by the major prognostic factors. As expected, uni- and multivariate analyses 
performed in this whole set of 498 tumors gave the same results than in the validation set (data not shown).

\section{DISCUSSION}

Refining the assessment of the metastatic risk after a given CT regimen and the decision regarding what regimen to use is one of the two major priorities for translational breast cancer research [62]. Using DNA microarrays, we profiled a multicentric series of 595 breast cancers, including 498 cases treated with adjuvant anthracycline-based CT without taxane. To our knowledge, this is the largest expression profiling study that specifically addresses the issue of survival prediction in breast cancer after a specific regimen of adjuvant therapy.

In patients treated with anthracycline-based CT without taxane, our predictor identified a "good-prognosis group" ( $75 \%$ of patients) with $82 \% 5$-year MFS and a "poor-prognosis group" ( $25 \%$ of patients) with 56\% 5-year MFS. The strength of our results relies on several points: the large samples series, representative of the population treated with adjuvant $\mathrm{CT}$, the homogeneity of adjuvant $\mathrm{CT}$, the use of a learning set and a totally independent validation set, the profiling on two different technological platforms, and the similar performances of our multigene predictor in the learning and the validation sets. We first identified a genomic predictor of MFS after CT in a retrospective multicentric series of 323 samples: the HR for metastatic relapse was 2.35 between the two predictor-based patients groups, with an overall predictive accuracy of $77 \%$. The robustness of the predictor was confirmed in the validation set of 
175 similarly treated samples with $74 \%$ of accurate prediction and a resulting $\mathrm{HR}$ of 3.18. Importantly, $73 \%$ of patients from the validation set were treated in the reference arm of a prospective multicentric clinical trial [13] and with heterogeneous conditions of sample acquisition and handling across 17 different hospitals. Two different microarray platforms were used for the validation set. All these potential sources of heterogeneity did not alter the performance of our predictor. The predictor outperformed the individual current prognostic factors in multivariate analysis, both in the learning and the validation sets, as well as the NPI prognostic classifier, and added independent prognostic information.

Our predictor could help tailor the adjuvant CT regimens in patients candidate to CT. In the "good-prognosis group", sensitivity to anthracyclines and/or weak metastatic propensity may explain the favorable survival. As compared with historical data of untreated patients the benefit of adjuvant CT appears substantial $[63,64]$, suggesting that these patients remain candidates for adjuvant anthracycline-based CT, with or without taxane. Conversely and as compared with data of untreated patients $[63,64]$, the benefit of anthracycline-based CT without taxane in the "poor-prognosis group" appears weak $\mathrm{A}$ different $\mathrm{CT}$ regimen (without anthrcayclines) or the testing of new drugs in clinical trials should be considered in this group.

In the adjuvant setting, the onset of metastasis depends on several factors, including sensitivity to systemic therapy but also intrinsic disease aggressiveness. At the biological level, our predictor translates a complex interaction between factors that govern natural history of disease and chemosensitivity. Whether the included genes are causative or predictive of the phenotype or reflect another associated phenomenon remains to be explored. Several genes have a known biological or clinical relevance in breast cancer, such as ESR1, BCL2, TP53, PLAT and MAPT. Although individual 
genes may have functional relevance, the coordinated expression of several gene families and cell processes is likely to be the most important determinant of outcome. This was well evidenced by the statistical significance of the three predictor metagenes that we observed in multivariate analysis, but not in univariate analysis. The under-ER and under-PR metagenes, which overall scored high in "poor-prognosis tumors", comprised genes associated with proliferation (AURKA, CDK4,CDKL5, cyclin genes, ENO1), stroma (ITGB3, proteases MMP7,15, CTSC, PLAT), immune response (IGHG1, interleukin receptors, CD300C) and basal phenotype (S100A6,A8, SFRP1, $L A M B 1)$. Conversely, the under-EGFR metagene, which overall scored high in "goodprognosis tumors", was rather associated with the luminal phenotype (ESR1, KRT18, GATA3, XBP1, TFF1-3, SCUBE2). Some genes and/or pathways included in our predictor might be used as targets for specific therapies.

\section{CONCLUSIONS}

Our predictor is a first step toward optimal selection of the most likely beneficial adjuvant CT regimen. Additional steps are required regarding its validation. The first one will be to confirm prospectively the MFS of the two groups of patients. We have recently launched a prospective study in "good-prognosis" N+ patients [65]. If validated, this predictor would allow moving away from the "one shoe fits all" strategy used in oncology for many years [66] and from the ongoing "therapeutical escalation". Such an issue is complementary to that addressed for $\mathrm{N}$ - patients in the ongoing MINDACT and TAILORx trials [34]. Finally, because our predictor is also relevant in N- early breast 
cancer, it might be useful for tailoring the adjuvant CT regimens in those patients defined as candidates for $\mathrm{CT}$.

\section{ACKNOWLEDGEMENTS}

We are grateful to L. Xerri and C. Mawas for encouragements. We thank F. Bonnet, S. Mathoulin-Pélissier, V. Brouste for their technical assistance and S. Deraco for his help in data analysis. This work was supported in part by Inserm, Institut PaoliCalmettes, and grants from Programme Hospitalier de Recherche Clinique 2001 (PHRC № 24-01 FB) and 2003 (№ 24-01 FB), Ligue Nationale Contre le Cancer (label DB), Association pour la Recherche sur le Cancer, Fédération Nationale des Centres de Lutte Contre le Cancer and Institut National du Cancer (ACl2004, PL2006, recherche translationnelle). The Biological Resource Centres in Oncology at Institut PaoliCalmettes and Centre Léon Bérard were supported by grants "Tumorothèques" from the French Ministry of Health and INCa, by grants "Collections 2003" from Inserm and the French Ministry of Research, and by grants "CEBS 2006" from the Agence Nationale de la Recherche (ANR).

\section{CONFLICT OF INTEREST}

Ipsogen employees: N Borie, JM Le Doussal, S Debono, A Martinec, F Hermitte, V Fert. Ipsogen shareholders: S Debono, F Hermitte, V Fert. The other authors declare they have no competing interest.

\section{REFERENCES}


1. Group EBCTCI (2005) Effects of chemotherapy and hormonal therapy for early breast cancer on recurrence and 15-year survival: an overview of the randomized trials. Lancet 365:1687-1717.

2. Piccart-Gebhart MJ, Procter M, Leyland-Jones B et al (2005) Trastuzumab after adjuvant chemotherapy in HER2-positive breast cancer. N Engl J Med 353:1659-1672

3. Gonzalez-Angulo AM, Hortobagyi GN, Esteva FJI (2006) Adjuvant Therapy with Trastuzumab for HER-2/neu-Positive Breast Cancer. Oncologist 11:857-867

4. Romond EH, Perez EA, Bryant $J$ et al (2005) Trastuzumab plus adjuvant chemotherapy for operable HER2-positive breast cancer. N Engl J Med 353:1673-1684 5. Sparano JA, Hortobagyi GN, Gralow JR, Perez EA, Comis RLI (2010) Recommendations for research priorities in breast cancer by the Coalition of Cancer Cooperative Groups Scientific Leadership Council: systemic therapy and therapeutic individualization. Breast Cancer Res Treat 119:511-527

6. Goldhirsch A, Glick JH, Gelber RD, Coates AS, Senn HJI (2001) Meeting highlights: International Consensus Panel on the Treatment of Primary Breast Cancer. Seventh International Conference on Adjuvant Therapy of Primary Breast Cancer. J Clin Oncol 19:3817-3827

7. Colozza M, de Azambuja E, Cardoso F, Bernard C, Piccart MJI (2006) Breast cancer: achievements in adjuvant systemic therapies in the pre-genomic era. Oncologist 11:111-125

8. Piccart MJ, de Valeriola D, Dal Lago L et al (2005) Adjuvant chemotherapy in 2005: standards and beyond. Breast 14:439-445

9. Buzdar AU, Singletary SE, Valero V et al (2002) Evaluation of paclitaxel in adjuvant chemotherapy for patients with operable breast cancer: preliminary data of a prospective randomized trial. Clin Cancer Res 8:1073-1079

10. Henderson IC, Berry DA, Demetri GD et al (2003) Improved outcomes from adding sequential Paclitaxel but not from escalating Doxorubicin dose in an adjuvant chemotherapy regimen for patients with node-positive primary breast cancer. J Clin Oncol 21:976-983.

11. Mamounas EP, Bryant J, Lembersky B et al (2005) Paclitaxel after doxorubicin plus cyclophosphamide as adjuvant chemotherapy for node-positive breast cancer: results 
from NSABP B-28. J Clin Oncol 23:3686-3696.

12. Martin M, Pienkowski T, Mackey J et al (2005) Adjuvant docetaxel for node-positive breast cancer. N Engl J Med 352:2302-2313

13. Roche H, Fumoleau P, Spielmann M et al (2006) Sequential adjuvant Epirubicinbased and Docetaxel Chemotherapy for node-positive breast cancer patients: the PACS01 trial. J Clin Oncol 24:5664-5671

14. Hayes DFI (2005) Prognostic and predictive factors revisited. Breast 14:493-499

15. Hayes DFI (2009) is there a standard type and duration of adjuvant chemotherapy for early stage breast cancer? Breast 18 Suppl 3:S131-134

16. Bertucci F, Finetti P, Cervera N et al (2006) Gene expression profiling and clinical outcome in breast cancer. Omics 10:429-443

17. Sorlie T, Perou CM, Tibshirani R et al (2001) Gene expression patterns of breast carcinomas distinguish tumor subclasses with clinical implications. Proc Natl Acad Sci U S A 98:10869-10874

18. Sorlie T, Tibshirani R, Parker J et al (2003) Repeated observation of breast tumor subtypes in independent gene expression data sets. Proc Natl Acad Sci U S A 100:8418-8423

19. van de Vijver MJ, He YD, van't Veer LJ et al (2002) A gene-expression signature as a predictor of survival in breast cancer. N Engl J Med 347:1999-2009.

20. van 't Veer LJ, Dai H, van de Vijver MJ et al (2002) Gene expression profiling predicts clinical outcome of breast cancer. Nature 415:530-536

21. Wang Y, Klijn JG, Zhang Y et al (2005) Gene-expression profiles to predict distant metastasis of lymph-node-negative primary breast cancer. Lancet 365:671-679.

22. Foekens JA, Atkins D, Zhang $Y$ et al (2006) Multicenter validation of a gene expression-based prognostic signature in lymph node-negative primary breast cancer. $\mathrm{J}$ Clin Oncol 24:1665-1671

23. Buyse M, Loi S, van't Veer L et al (2006) Validation and clinical utility of a 70-gene prognostic signature for women with node-negative breast cancer. J Natl Cancer Inst 98:1183-1192

24. Desmedt C, Piette F, Loi S et al (2007) Strong time dependence of the 76-gene prognostic signature for node-negative breast cancer patients in the TRANSBIG multicenter independent validation series. Clin Cancer Res 13:3207-3214 
25. Sotiriou C, Wirapati $P$, Loi $S$ et al (2006) Gene expression profiling in breast cancer: understanding the molecular basis of histologic grade to improve prognosis. $J$ Natl Cancer Inst 98:262-272

26. Ivshina AV, George J, Senko O et al (2006) Genetic reclassification of histologic grade delineates new clinical subtypes of breast cancer. Cancer Res 66:10292-10301

27. Miller LD, Smeds J, George J et al (2005) An expression signature for p53 status in human breast cancer predicts mutation status, transcriptional effects, and patient survival. Proc Natl Acad Sci U S A 102:13550-13555

28. Naderi A, Teschendorff AE, Barbosa-Morais NL et al (2007) A gene-expression signature to predict survival in breast cancer across independent data sets. Oncogene 26:1507-1516

29. Ma XJ, Wang Z, Ryan PD et al (2004) A two-gene expression ratio predicts clinical outcome in breast cancer patients treated with tamoxifen. Cancer Cell 5:607-616.

30. Paik S, Shak S, Tang G et al (2004) A multigene assay to predict recurrence of tamoxifen-treated, node-negative breast cancer. N Engl J Med 351:2817-2826

31. Oh DS, Troester MA, Usary J et al (2006) Estrogen-regulated genes predict survival in hormone receptor-positive breast cancers. J Clin Oncol 24:1656-1664

32. Loi S, Haibe-Kains B, Desmedt C et al (2007) Definition of clinically distinct molecular subtypes in estrogen receptor-positive breast carcinomas through genomic grade. J Clin Oncol 25:1239-1246

33. Teschendorff AE, Naderi A, Barbosa-Morais NL et al (2006) A consensus prognostic gene expression classifier for ER positive breast cancer. Genome Biol 7:R101

34. Sotiriou C, Piccart MJI (2007) Taking gene-expression profiling to the clinic: when will molecular signatures become relevant to patient care? Nat Rev Cancer 7:545-553

35. Ross JS, Hatzis C, Symmans WF, Pusztai L, Hortobagyi GNI (2008) Commercialized multigene predictors of clinical outcome for breast cancer. Oncologist 13:477-493

36. Ayers M, Symmans WF, Stec J et al (2004) Gene expression profiles predict complete pathologic response to neoadjuvant paclitaxel and fluorouracil, doxorubicin, and cyclophosphamide chemotherapy in breast cancer. J Clin Oncol 22:2284-2293.

37. Bertucci F, Finetti $P$, Rougemont $J$ et al (2004) Gene expression profiling for 
molecular characterization of inflammatory breast cancer and prediction of response to chemotherapy. Cancer Res 64:8558-8565

38. Chang JC, Wooten EC, Tsimelzon A et al (2003) Gene expression profiling for the prediction of therapeutic response to docetaxel in patients with breast cancer. Lancet 362:362-369.

39. Hannemann J, Oosterkamp HM, Bosch CA et al (2005) Changes in gene expression associated with response to neoadjuvant chemotherapy in breast cancer. $\mathrm{J}$ Clin Oncol. 23:3331-3342.

40. Bonnefoi $\mathrm{H}$, Potti A, Delorenzi $M$ et al (2007) Validation of gene signatures that predict the response of breast cancer to neoadjuvant chemotherapy: a substudy of the EORTC 10994/BIG 00-01 clinical trial. Lancet Oncol 8:1071-1078

41. Folgueira MA, Carraro DM, Brentani $\mathrm{H}$ et al (2005) Gene expression profile associated with response to doxorubicin-based therapy in breast cancer. Clin Cancer Res 11:7434-7443

42. Hess KR, Anderson K, Symmans WF et al (2006) Pharmacogenomic predictor of sensitivity to preoperative chemotherapy with paclitaxel and fluorouracil, doxorubicin, and cyclophosphamide in breast cancer. J Clin Oncol 24:4236-4244

43. Iwao-Koizumi K, Matoba $\mathrm{R}$, Ueno $\mathrm{N}$ et al (2005) Prediction of docetaxel response in human breast cancer by gene expression profiling. J Clin Oncol 23:422-431

44. Farmer $\mathrm{P}$, Bonnefoi $\mathrm{H}$, Anderle $\mathrm{P}$ et al (2009) A stroma-related gene signature predicts resistance to neoadjuvant chemotherapy in breast cancer. Nat Med 15:68-74 45. Bertucci F, Houlgatte R, Benziane A et al (2000) Gene expression profiling of primary breast carcinomas using arrays of candidate genes. Hum Mol Genet 9:29812991

46. Bertucci F, Nasser V, Granjeaud S et al (2002) Gene expression profiles of poorprognosis primary breast cancer correlate with survival. Hum Mol Genet 11:863-872.

47. Specht K, Harbeck N, Smida J et al (2009) Expression profiling identifies genes that predict recurrence of breast cancer after adjuvant CMF-based chemotherapy. Breast Cancer Res Treat 118:45-56

48. Campone M, Campion L, Roche $\mathrm{H}$ et al (2008) Prediction of metastatic relapse in node-positive breast cancer: establishment of a clinicogenomic model after FEC100 adjuvant regimen. Breast Cancer Res Treat 109:491-501 
49. Pawitan Y, Bjohle J, Amler L et al (2005) Gene expression profiling spares early breast cancer patients from adjuvant therapy: derived and validated in two populationbased cohorts. Breast Cancer Res 7:R953-964

50. Jezequel $P$, Campone $M$, Roche $\mathrm{H}$ et al (2009) A 38-gene expression signature to predict metastasis risk in node-positive breast cancer after systemic adjuvant chemotherapy: a genomic substudy of PACS01 clinical trial. Breast Cancer Res Treat 116:509-520

51. Bertucci F, Borie N, Ginestier C et al (2004) Identification and validation of an ERBB2 gene expression signature in breast cancers. Oncogene 23:2564-2575.

52. Vey N, Mozziconacci MJ, Groulet-Martinec A et al (2004) Identification of new classes among acute myelogenous leukaemias with normal karyotype using gene expression profiling. Oncogene 23:9381-9391.

53. Bertucci F, Orsetti B, Negre V et al (2008) Lobular and ductal carcinomas of the breast have distinct genomic and expression profiles. Oncogene 27:5359-5372

54. Kaplan EL, Meier PI (1958) Non-parametric estimation for incomplete observation. J Am Stat Assoc 53:457-481

55. Cox DRI (1972) Regression models and life table. J R Stat Soc $[B]$ 34:187-220

56. Todd JH, Dowle C, Williams MR et al (1987) Confirmation of a prognostic index in primary breast cancer. $\mathrm{Br} \mathrm{J}$ Cancer 56:489-492

57. Gruvberger S, Ringner M, Chen $Y$ et al (2001) Estrogen receptor status in breast cancer is associated with remarkably distinct gene expression patterns. Cancer Res 61:5979-5984.

58. Perou CM, Sorlie T, Eisen MB et al (2000) Molecular portraits of human breast tumours. Nature 406:747-752

59. West M, Blanchette C, Dressman $\mathrm{H}$ et al (2001) Predicting the clinical status of human breast cancer by using gene expression profiles. Proc Natl Acad Sci U S A 98:11462-11467

60. Bertucci F, Finetti P, Cervera N et al (2006) Gene expression profiling shows medullary breast cancer is a subgroup of basal breast cancers. Cancer Res 66:46364644

61. Desmedt C, Haibe-Kains B, Wirapati P et al (2008) Biological processes associated with breast cancer clinical outcome depend on the molecular subtypes. Clin 
Cancer Res 14:5158-5165

62. Dowsett M, Goldhirsch A, Hayes DF et al (2007) International Web-based consultation on priorities for translational breast cancer research. Breast Cancer Res 9:R81

63. Bonadonna G, Valagussa P, Moliterni A, Zambetti M, Brambilla Cl (1995) Adjuvant cyclophosphamide, methotrexate, and fluorouracil in node-positive breast cancer: the results of 20 years of follow-up. N Engl J Med 332:901-906

64. Demicheli R, Miceli R, Brambilla C et al (1999) Comparative analysis of breast cancer recurrence risk for patients receiving or not receiving adjuvant cyclophosphamide, methotrexate, fluorouracil (CMF). Data supporting the occurrence of 'cures'. Breast Cancer Res Treat 53:209-215

65. Bertucci F, Ferrero JM, Bachelot T et al (2009) SA02 trial: a genomics-based prospective study of adjuvant chemotherapy in node-positive early breast cancer with « good-prognosis signature ». SABCS 2009

66. Piccart-Gebhart MJI (2005) Moving away from the "one shoe fits all" strategy: the key to future progress in chemotherapy. J Clin Oncol 23:1611-1613 


\section{FIGURE LEGENDS}

Figure 1. Hierarchical clustering of tumors treated with adjuvant chemotherapy. A/ Hierarchical clustering of 468 samples and 2.261 filtered clones, based on expression data generated with Ipsogen microarrays. Each row represents a gene and each column a sample. The expression level of each gene in each sample is relative to its median abundance across the samples and is depicted according to the color scale shown. Red and green indicate expression levels respectively above and below the median. Colored branches in the dendrogram of genes and colored bars in the left column to the right of the matrix indicate the locations of gene clusters of interest zoomed in $\mathrm{C}$. Black and grey bars in the right column to the right of the matrix indicate the location of gene clusters selected to define metagenes from clustering: bars with an * show the 12 clusters entered in the predictor building (from top to bottom, metagenes $141,171,144,51,448,1001,27,310,240,48,187$, and 66). Other bars correspond (from top to bottom) to metagenes $678,875,242,8$, and 9 not retained to build the predictor. B/Dendrogram and histoclinical features of samples. Top, two large groups of samples (left and right) are evidenced and delimited by the orange vertical line. Bottom, some relevant features of samples are represented according to a color ladder (unavailable, oblique feature): ER, PR, ERBB2 IHC status (negative, white; positive, black), SBR grade (I, white; II, grey; III, black), and metastatic relapse during follow-up (no, white; yes, black). C/Expanded view of selected gene clusters named from top to bottom: luminal/ER (dark blue bar), proliferation (dark grey), early response (dark green), stromal (orange), ERBB2-related (pink), basal (red), immune (light green). Some genes (EntrezGene symbol) included in each cluster are listed. 
Figure 2: Classification of tumors treated with adjuvant chemotherapy based on the genomic predictor and survival.

Correlation between the classification of 498 tumors based on the genomic predictor and the occurrence of metastatic relapse in the learning set of samples $(A)$ and in the validation set (B). Left, cross-table; Right, Kaplan-Meier metastasis-free survival curves. HR means hazard ratio for metastasis in the "poor-prognosis group" as compared to the "good-prognosis group". 
Table 1: Samples from 498 patients treated with adjuvant chemotherapy and used for identification and validation of the genomic predictor.

Table 2: Histoclinical characteristics of 498 patients treated with adjuvant chemotherapy, according to the membership to the "good-prognosis" or the "poor-prognosis group" as defined using the genomic predictor.

Table 3: Cox proportional-hazards univariate and multivariate analyses in metastasis-free survival in the learning and the validation sets of patients treated with adjuvant chemotherapy. 


\section{SUPPLEMENTARY FILES}

\section{Supplementary text}

Supplementary Figure 1: Kaplan-Meier MFS for patients treated with adjuvant anthracycline-based chemotherapy. A/MFS for all 498 patients. $B-D /$ MFS in the "good-prognosis group" or the "poor-prognosis group" defined using the genomic predictor; $B /$ MFS for all 498 patients; $C / M F S$ for 417 patients with $\mathrm{N}$ + cancer; $D /$ MFS for 80 patients with $\mathrm{N}$ - cancer. HR means hazard ratio for metastasis in the "poorprognosis group" as compared to the "good-prognosis group".

Supplementary Table 1: Description and performance of phenotypic gene expression signatures identified by supervised analysis (MaxT method).

Supplementary Table 2: Phenotypic gene expression signatures associated with ER, PR, ERBB2, EGFR and Ki67 identified by supervised MaxT method.

Supplementary Table 3: Univariate-multivariate Cox analysis of 19 metagenes for metastasis-free survival (MFS) in 323 patients from the learning set.

Supplementary Table 4: Histoclinical data of the 595 tumors profiled. 


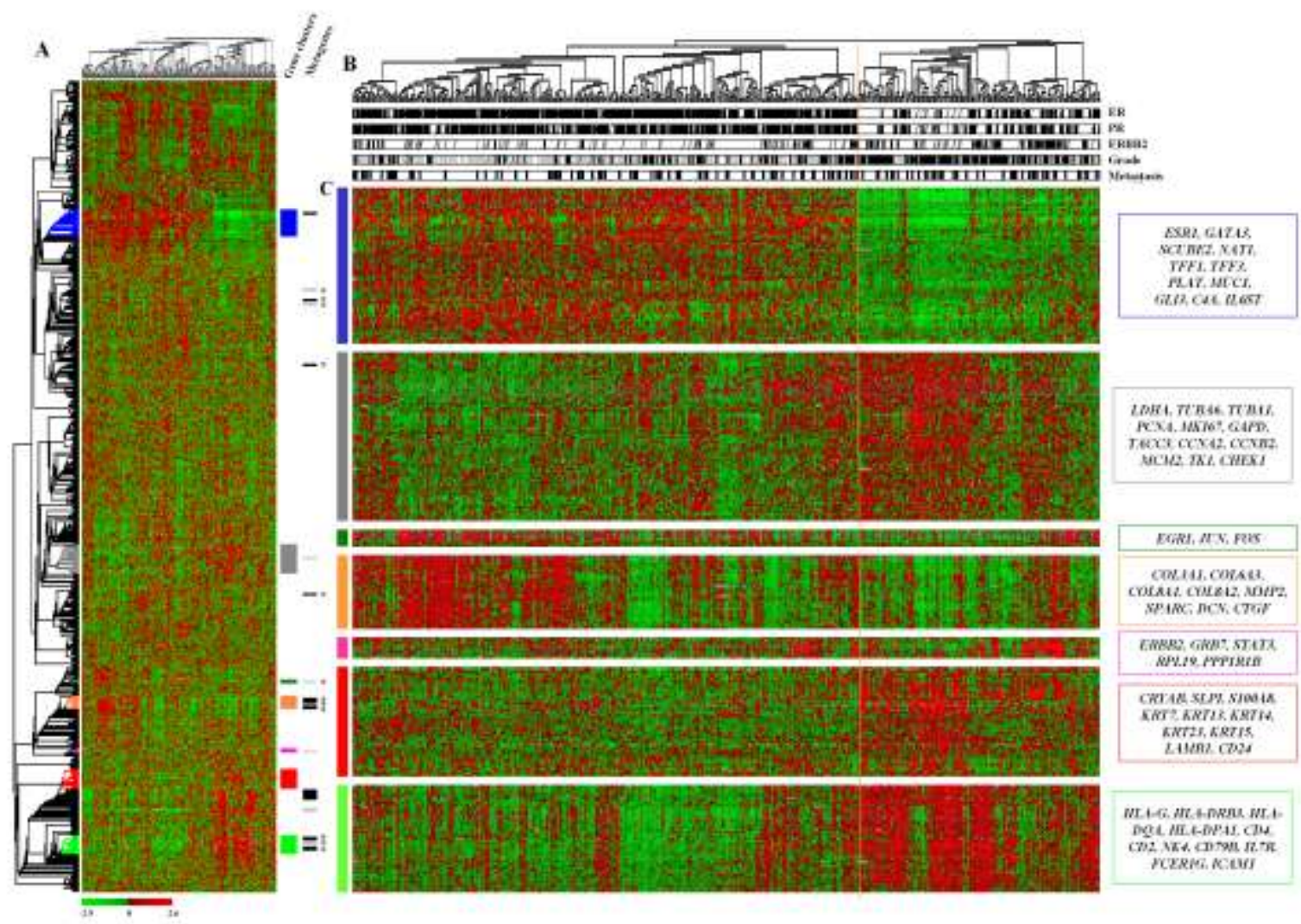

$\mathbf{A}$

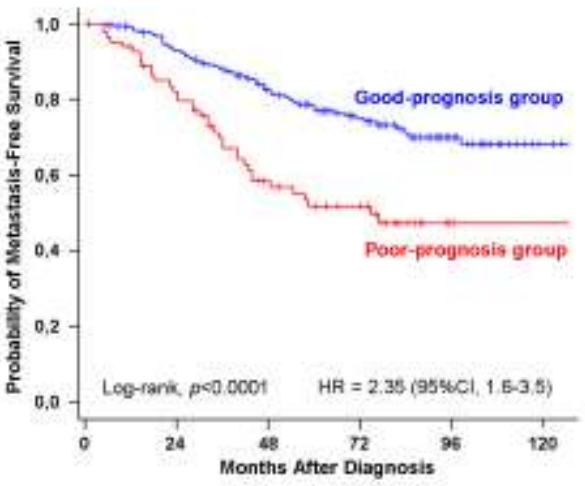

B

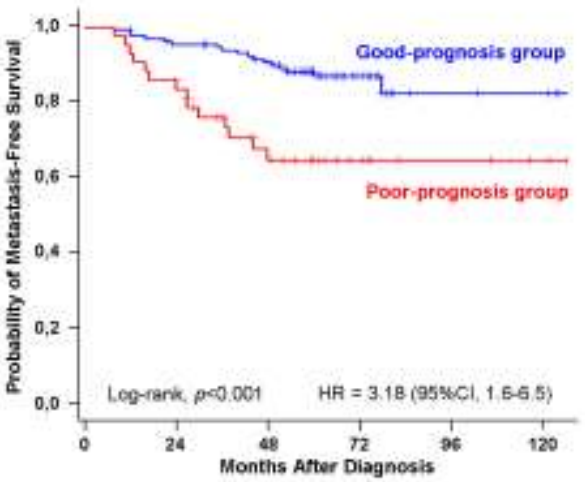

Table 1: Samples from 498 patients treated with adjuvant chemotherapy and used for identification and validation of the genomic predictor. 


\begin{tabular}{|c|c|c|c|}
\hline Institution & $\begin{array}{c}\text { No. of } \\
\text { samples }\end{array}$ & $\begin{array}{c}\text { Microarray } \\
\text { platform }\end{array}$ & $\begin{array}{c}\text { Use in this } \\
\text { study }\end{array}$ \\
\hline Paoli-Calmettes & 213 & \multirow{4}{*}{$\begin{array}{c}\text { Ipsogen } \\
\text { DiscoveryChip }\end{array}$} & Learning \\
\hline Léon Bérard & 110 & & set \\
\hline Bergonié & 17 & & \multirow{3}{*}{$\begin{array}{c}\text { Validation } \\
\text { set }\end{array}$} \\
\hline PACS01, A arm & 128 & & \\
\hline Paoli-Calmettes & 30 & $\begin{array}{c}\text { Affymetrix } \\
\text { U133 Plus } 2.0\end{array}$ & \\
\hline
\end{tabular}

Table 2: Histoclinical characteristics of 498 patients treated with adjuvant chemotherapy, according to the membership to the "good-prognosis" or the "poor-prognosis group" as defined using the genomic predictor

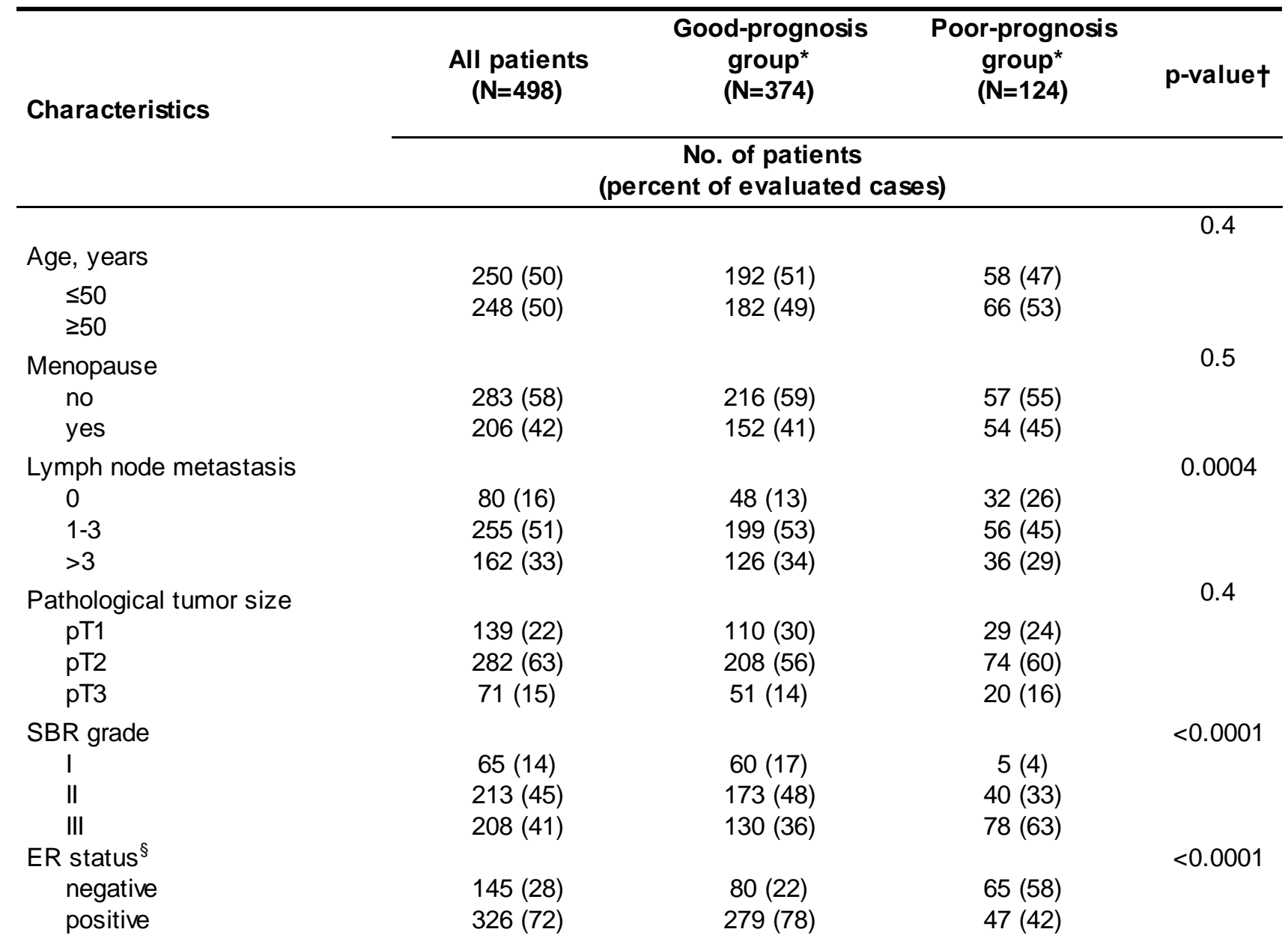




\begin{tabular}{|c|c|c|c|c|}
\hline PR status ${ }^{\S}$ & & & & $<0.0001$ \\
\hline negative & $182(36)$ & $113(31)$ & $69(61)$ & \\
\hline positive & $291(64)$ & $246(69)$ & $45(39)$ & \\
\hline ERBB2 status ${ }^{\S}$ & & & & 0.6 \\
\hline negative & $331(85)$ & $251(85)$ & $80(88)$ & \\
\hline positive & $54(15)$ & $43(15)$ & $11(12)$ & \\
\hline EGFR status ${ }^{\S}$ & & & & 1 \\
\hline negative & $114(77)$ & $89(77)$ & $25(76)$ & \\
\hline positive & $35(23)$ & $27(23)$ & $8(24)$ & \\
\hline Ki67 status ${ }^{\S}$ & & & & 0.0002 \\
\hline negative & $62(50)$ & $57(53)$ & $5(16)$ & \\
\hline positive & 77 (50) & $50(47)$ & $27(84)$ & \\
\hline Follow-up ${ }^{\dagger+}$, months & & & & 1 \\
\hline median (range) & $68(1,175)$ & $68(1,175)$ & $68(7,155)$ & \\
\hline 5-year MFS & & & & $<0.0001$ \\
\hline$\%[95 \% \mathrm{Cl}]$ & 76 [71.7 - 79.7] & $82[77.8-86.1]$ & 56.[47.5 - 66.4] & \\
\hline
\end{tabular}

${ }^{*}$, as defined using the genomic predictor;

$\dagger, p$-values were calculated using the Chi-2 test for the comparison of numbers of patients, using the log-rank test for the comparison of metastasis-free survival (MFS), and using the Student T-test for the comparison of follow-up;

$\S$, protein expression status was measured in each center using $\mathrm{IHC}$ on whole sections using the following positivity cut-offs: $\geq 10 \%$ for ER (estrogen receptor), PR (progesterone receptor), and EGFR (epidermal growth factor receptor), $\geq 20 \%$ for Ki67 and 3+ for ERBB2 (HercepTest)

††calculated, for the 369 patients who did not experience metastatic relapse, from the date of diagnosis to the time of last follow-up;

Note: SBR, Scarff-Bloom-Richardson; Cl, confidence interval.

Table 3: Cox proportional-hazards univariate and multivariate analyses in metastasis-free survival in the learning and the validation sets of patients treated with adjuvant chemotherapy. 


\section{Sample set \\ Learning set Genomic group - Poor-prognosis vs Good-prognosis}

$$
\text { Age - > } 50 \text { vs } \leq 50
$$

Menopause - yes vs no

Lymph node metastasis - $>3$ vs $\leq 3$

Pathological tumor size- $\mathrm{pT} 2-3$ vs $\mathrm{pT} 1$

SBR Grade - 2-3 vs 1

ER status - pos vs neg

PR status - pos vs neg
Univariate analysis

HR [95\% Cl] p-value

$2.3[1.6-3.5]<0.0001$

$1.2[0.8-1.8] \quad 0.40$

$1.1[0.7-1.6] \quad 0.68$

$1.5[1-2.3]$

0.046

$1.1[0.7-1.7]$

0.79

$2.4[1.1-5.1]$

0.025

$0.9[0.6-1.4]$

$0.7[0.4-1]$

0.55

0.05

$1[0.5-2]$
Multivariate analysis

\begin{tabular}{cc} 
HR [95\%Cl] & p-value \\
$2.3[1.47-3.49]$ & 0.00021 \\
--- & --- \\
--- & --- \\
$1.6[1.02-2.39]$ & 0.041 \\
--- & --- \\
NS & NS \\
--- & --- \\
NS & NS \\
--- & --- \\
\hline
\end{tabular}

$3.2[1.6-6.5] \quad 0.0008$

$4.5[2.12-9.71] \quad<0.0001$

$1.2[0.6-2.3] \quad 0.68$

$1.1[0.5-2.4] \quad 0.76$

Menopause - yes vs no

Lymph node metastasis - $>3$ vs $\leq 3$

Pathological tumor size- $\mathrm{pT2}-3$ vs $\mathrm{pT} 1$

SBR Grade - 2-3 vs 1

ER status - pos vs neg

PR status - pos vs neg

ERBB2 status - pos vs neg
3 [1.5-6.1] $\quad 0.0017$

$2.4[1-6] \quad 0.048$

$--\quad 0.0138$

$0.5[0.2-1] \quad 0.046$

$0.7[0.4-1.5] \quad 0.37$

$2.8[1.2-6.2] \quad 0.0099$

$\begin{array}{cc}--- & --- \\ --- & -- \\ 2.8[1.30-5.93] & 0.0085 \\ \text { NS } & \text { NS } \\ \text { NS } & \text { NS } \\ \text { NS } & \text { NS } \\ --- & --- \\ \text { NS } & \text { NS }\end{array}$

\section{Validation set Genomic group - Poor-prognosis vs Good-prognosis} NPI classifier - high-risk vs not high risk

$3.2[1.6-6.5] \quad 0.0008$

$3.5[1.61-7.45]$

0.001

$2.8[1.3-6.3] \quad 0.0076$

$2.4[1.08-5.47]$ 0.03 